\title{
ON CORRELATIONS BETWEEN LOCAL MICROSTRUCTURE AND THE APPEARANCE OF FIBRE BREAKS IN UNIDIRECTIONAL COMPOSITE MATERIALS
}

\author{
Christian Breite $^{1^{*}}$, Stepan V. Lomov ${ }^{1}$, Yentl Swolfs ${ }^{1}$ \\ ${ }^{1}$ Department of Materials Engineering, KU Leuven, \\ Kasteelpark Arenberg 44 box 2450, 3001 Leuven, Belgium \\ * christian.breite@kuleuven.be
}

The recent development of a high precision fibre segmentation method by Emerson et al. [1] enabled the automated identification of fibre breaks in unidirectional composites (Breite et al. [2]). It is now possible to investigate the correlation of microstructural parameters of the fibre trajectories with the formation of fibre breaks and clusters of fibre breaks. The dataset used in the present study is based on the fibre trajectories segmented from a volume of $\sim 0.36 \mathrm{~mm}^{3}$, taken out from a flat sample, in-situ loaded under synchrotron radiation computed tomography to a tensile stress corresponding to $91 \%$ of the ultimate tensile stress. It consists of $\sim 5000$ fibres, $\sim 9 \cdot 10^{5}$ fibre segments of $\sim 6.5 \mu \mathrm{m}$ length and 92 fibre breaks. Statistical comparisons have been performed for the local fibre volume fraction, fitted sinusoidal parameters as measures for the local fibre waviness and the local in-plane and out-of-plane fibre angles measured in each of the fibre segments. The statistical methodology relies on repeated resampling from large population down to the size of the sparsely populated fibre breaks data. Insights were gained on differences between distributions of the microstructure parameters near broken fibres vs. unbroken fibres, fibre break sites vs. intact regions and single fibre break sites vs. clustered fibre break sites (Figure 1). In contrast to earlier work by Rosini et al. [3], the present work demonstrates a previously unknown link between the local disturbance of the alignment angles and the formation of fibre break clusters. This result highlights a major drawback in most state-ofthe-art tensile failure models, which do not consider the micro-scale misalignment of the fibres. Incorporating the local fibre alignment in the next generation models will help to better align model predictions with observable material behaviour and hence facilitate the goal of blind prediction.

The research leading to these results has been conducted in the framework of the FiBreMoD project and has received funding from the European Union's Horizon 2020 research and innovation programme under the Marie Skłodowska-Curie grant agreement No 722626 .
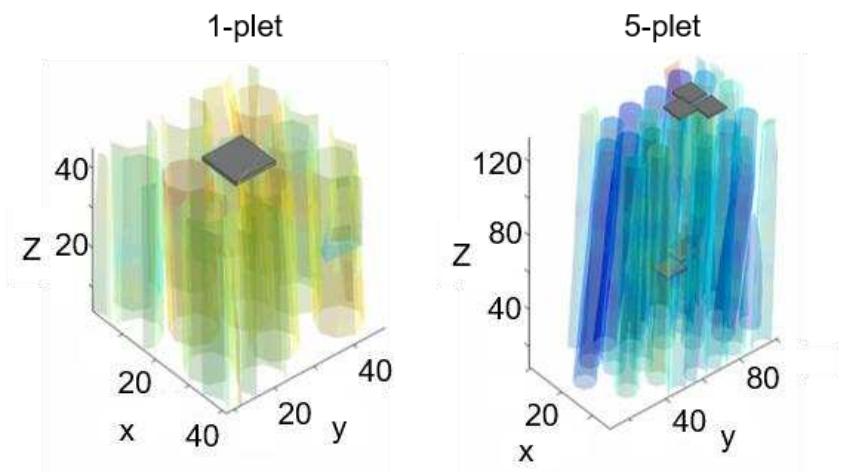

Local in-plane fibre angle

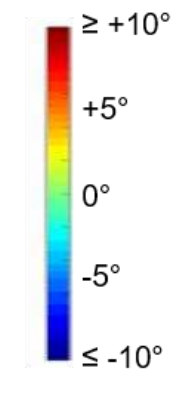

Figure 1. Microstructure near different fibre break configurations with transparent fibres colour coded with the local in-plane angle and fibre breaks visualised as grey squares. Coordinates are in px with $1 \mathrm{px}=650 \mathrm{~nm}$.

\section{References}

[1] M.J. Emerson, K.M. Jespersen, et al. (2017) Individual fibre segmentation from 3D X-ray computed tomography for characterising the fibre orientation in unidirectional composite materials. Composites Part A: Applied Science and Manufacturing, 97, 83-92.

[2] C. Breite, E. Schöberl, et al. (2021) 4D analysis of reconstructed fibres and fibre breaks in unidirectional composite materials Composites Part A: Applied Science and Manufacturing, in preparation.

[3] S. Rosini, M.N. Mavrogordato, et al. (2019) In situ statistical measurement of local morphology in carbon-epoxy composites using synchrotron X-ray computed tomography. Composites Part A: Applied Science and Manufacturing, 125, 105543. 